\title{
MANEJO INTEGRADO DE LA MARCHITEZ VASCULAR DEL TOMATE [Fusarium oxysporum f. sp. Iycopersici (SACC.) W.C. SNYDER \& H.N. HANSEN]: UNA REVISIÓN
}

\section{INTEGRATED DISEASE MANAGEMENT OF FUSARIUM WILT OF TOMATO [Fusarium oxysporum f. sp. Iycopersici (SACC.) W.C. SNYDER \& H.N. HANSEN]: A REVIEW}

\author{
Luisa Mayens Vásquez-Ramírez ${ }^{1}$, Jairo Castaño-Zapata ${ }^{2}$
}

${ }^{1}$ Ingeniera Agrónoma, c.M.Sc. Fitopatología, Facultad de Ciencias Agropecuarias. Universidad de Caldas, Calle 65 No. $26-$ 10, Manizales, Colombia, e-mail: luisamayens@gmail.com; ${ }^{2}$ Ingeniero Agrónomo, Fitopatólogo. Ph.D., Profesor Titular, Departamento de Producción Agropecuaria, Facultad de Ciencias Agropecuarias. Universidad de Caldas, Calle 65 No. 26-10, Manizales, Colombia, e-mail: jairo.castano_z@ucaldas.edu.co.

Rev. U.D.C.A Act. \& Div. Cient. 20(2): 363-374, Julio-Diciembre, 2017

\section{RESUMEN}

El tomate (Solanum lycopersicum L.) es una de las hortalizas más consumidas en el mundo y es atacada por numerosas enfermedades, entre las cuales, está la marchitez vascular, causada por Fusarium oxysporum f. sp. lycopersici. El patógeno, por ser habitante del suelo, es difícil de manejar. Una vez el suelo se infesta puede permanecer con el patógeno por tiempo indefinido. Debido al cultivo sucesivo de tomate en el mismo lugar, la población del hongo aumenta $y$, por ende, la incidencia de la enfermedad. Aún no se han establecido umbrales de daño económico ni sistemas de predicción, pero se conocen algunas condiciones del suelo, que pueden disminuir la incidencia de la enfermedad, como $\mathrm{pH}$ alto o una población alta de microorganismos antagonistas. Se han reportado numerosas prácticas de manejo enfocadas, especialmente hacia el suelo; sin embargo, son pocos los reportes acerca del manejo integrado de la enfermedad. Para la preparación de este documento, se revisó la literatura mundial y se analizó la naturaleza del patógeno, su ciclo de vida y diseminación, conocimiento primordial para realizar un manejo adecuado de la enfermedad; también las diferentes prácticas de manejo y, finalmente, la combinación de estas prácticas. El objetivo de esta revisión fue reunir la información más relevante generada en los últimos años, en torno al manejo integrado de F. oxysporum f. sp. lycopersici, enfocando cada práctica dentro del modelo logístico de Van der Plank, que permite darles una interpretación cuantitativa.

Palabras clave: Hyphomycetes, marchitamiento, hongos, protección, modelo logístico.

\section{SUMMARY}

Tomato (Solanum lycopersicum L.) is one of the most consumed vegetables in the world and is attacked by numerous diseases, including the vascular wilt, caused by Fusarium oxysporum f. sp. lycopersici. The pathogen, being an inhabitant of the soil is difficult to manage. Once the soil is infested, it can remain with the pathogen indefinitely. Due to the successive cultivation of tomato in the same field the population of the fungus increases and, consequently, the incidence of the disease. No thresholds for economic damage or prediction systems have yet been established, but some soil conditions are known to reduce the incidence of the disease, such as high $\mathrm{pH}$ or a high population of antagonistic microorganisms. Numerous management practices have been reported, especially focused toward the soil; however, there are few articles on integrated management of the disease. For the preparation of this document, the world literature was reviewed and addressed the nature of the pathogen, its life cycle and dissemination, essentials to carry out an adequate management of the disease; also, the different management practices and finally the combination of these practices. The objective of this review was to gather the most relevant information generated in recent years on the integrated management of F. oxysporum f. sp. lycopersici, focusing each practice within the logistic model of Van der Plank, which allows giving to each one a quantitative interpretation.

Key words: Hyphomycetes, tomato wilt, fungi, protection, logistic model. 


\section{INTRODUCCIÓN}

El tomate (Solanum lycopersicum L.) es una de las hortalizas más consumidas en el mundo, su producción en el 2014 fue de 170.750.767 t (FAOSTAT, 2014). Se cultiva en más de 100 países para consumo fresco e industrial; el $76 \%$ de la producción, se concentra en China, India, Estados Unidos, Turquía, Egipto, Irán, Italia, España, Brasil y México (FAOSTAT, 2014). El consumo promedio en países desarrollados es de $86 \mathrm{~g}$ diarios per capita y en vía de desarrollo, $27 \mathrm{~g}$. Colombia produjo 606.151t en el 2014, con un rendimiento promedio de 36,98t/ha. El rendimiento promedio mundial es de 33,98t/ha, cifra por debajo de la obtenida en áreas con mayor rendimiento y alta tecnificación, como Países Bajos, 505,618t/ha; Bélgica, 498,6t/ha; Reino Unido, 424,569t/ha y Noruega, con 362,18t/ha (FAOSTAT, 2014).

Su origen es América, lo que explica el elevado número de enemigos naturales en Colombia, que como es conocido, se convierten en plagas y en enfermedades, cuando se establecen monocultivos. Se reportan 48 enfermedades importantes, entre las cuales, está la marchitez vascular y el mal del talluelo, causados por Fusarium oxysporum f. sp. lycopersici (Sacc.) W.C. Snyder \& H.N. Hansen, una de las más de 100 formas especiales que se conocen de Fusarium oxysporum, de amplia distribución mundial y, del cual, no se conoce su teleomorfo (Gordon, 2017).

La enfermedad causa pérdidas entre 21 y $47 \%$ en cultivos a libre exposición y bajo cubierta (Ramyabharathi et al. 2012; Enespa \& Dwivedi, 2014); en Colombia, aún no se tienen datos de pérdidas por esta enfermedad. Cuando este patógeno ataca plántulas ocasiona mal del talluelo, que es favorecido por la carencia de lignina en el tallo, lo que las hace más susceptibles, permitiendo que el patógeno alcance rápidamente los vasos del xilema, causando la destrucción y el colapso del tejido (Agrios, 2005). El tejido vascular de una planta enferma se torna de color pardo oscuro, siendo más notable en el punto de unión del peciolo con el tallo. Este color es característico de la enfermedad y se emplea para su identificación; la médula permanece sana y, ocasionalmente, ocurre infección en el fruto, que se puede detectar por la decoloración del tejido vascular dentro de él (Jones, 1991).

Cuando el hongo ataca a plantas adultas, la enfermedad se conoce como marchitez vascular. Las plantas muestran amarillamiento, que comienza por las hojas bajeras y, por lo general, mueren (Figura 1A); la base del tallo adquiere un color oscuro (Figura 1C) y los haces vasculares se tornan de color pardo oscuro (Figura 1B). Una o varias ramas pueden mostrar síntomas; en ocasiones, las hojas presentan marchitez en los foliolos de un lado del pecíolo, mientras que los del lado opuesto se ven sanos. La marchitez del follaje es más notable después de la floración y cuajamiento de los frutos y durante los períodos más calurosos del día. Los síntomas son exacerbados por temperaturas altas, alrededor de $28^{\circ} \mathrm{C}$, por $\mathrm{pH}$ bajo del suelo y uso de fertilizantes amoniacales (Mc Govern \& Datnoff, 1992).

El hongo produce clamidosporas, microconidios y macroconidios (Figura 1D). Los macroconidios se han relacionado con la diseminación aérea, lo que sugiere una fase policíclica, no común en patógenos habitantes del suelo (Katan et al. 1997). El micelio sobrevive en residuos vegetales, como saprófito y en hospedantes alternos. Las clamidosporas permiten al hongo sobrevivir por largo tiempo; se producen a partir de la modificación de hifas o células conidiales. La formación de clamidosporas está relacionada con factores de estrés, como la ausencia del hospedante, agotamiento de nutrientes y ambientes adversos (Smith, 2007). Las clamidosporas germinan en condiciones favorables, incluyendo la presencia de exudados radicales; se ha demostrado que las clamidosporas resisten temperaturas altas y sobreviven más tiempo en el suelo que los conidios, además que causan síntomas más severos que los microconidios (Mc Govern, 2015).

La forma especial (f. sp.) lycopersici infecta solo a plantas de tomate susceptibles (Inami et al. 2014); no obstante, Fassihiani (2000) comprobó que esta forma especial es capaz de colonizar las raíces de plantas pertenecientes a otros géneros, como Oryzopsis, Digitaria, Amaranthus y Malva; además de plantas de zanahoria, trigo, varias especies de pastos, berenjena y ají. Según este investigador, aunque el patógeno puede colonizar a varias especies, solo se observaron síntomas de retraso en el crecimiento, no de marchitez, en algunas especies del género Malva, en berenjena y ají, estas últimas pertenecientes a la familia Solanaceae.

El hongo también sobrevive en el suelo, como saprófito o en restos vegetales, donde se mantiene viable hasta 10 años (Kant et al. 2011). Cuando una planta sana crece en suelo infestado por el hongo, el contacto con las raíces induce la germinación de los conidios, el tubo germinativo de la espora o el micelio penetra directamente por el ápice de las raíces o ingresa a éstas, a través de heridas o de los puntos de formación de las raíces laterales. El micelio avanza por medio del córtex de las raíces intercelularmente y cuando alcanza los vasos del xilema entra por los extremos. El micelio permanece en los vasos y se trasloca a través de ellos, principalmente, hacia arriba, hacia el tallo y la corona de la planta (Agrios, 2005). En los vasos, el micelio se ramifica y produce microconidios, que son liberados y llevados hacia el ápice en la corriente de la savia. Los microconidios germinan en el punto en donde el movimiento se detiene, el micelio penetra la pared superior del vaso y se producen más microconidios en el próximo vaso. El micelio también avanza lateralmente, dentro de los vasos adyacentes, penetrando por los extre- 


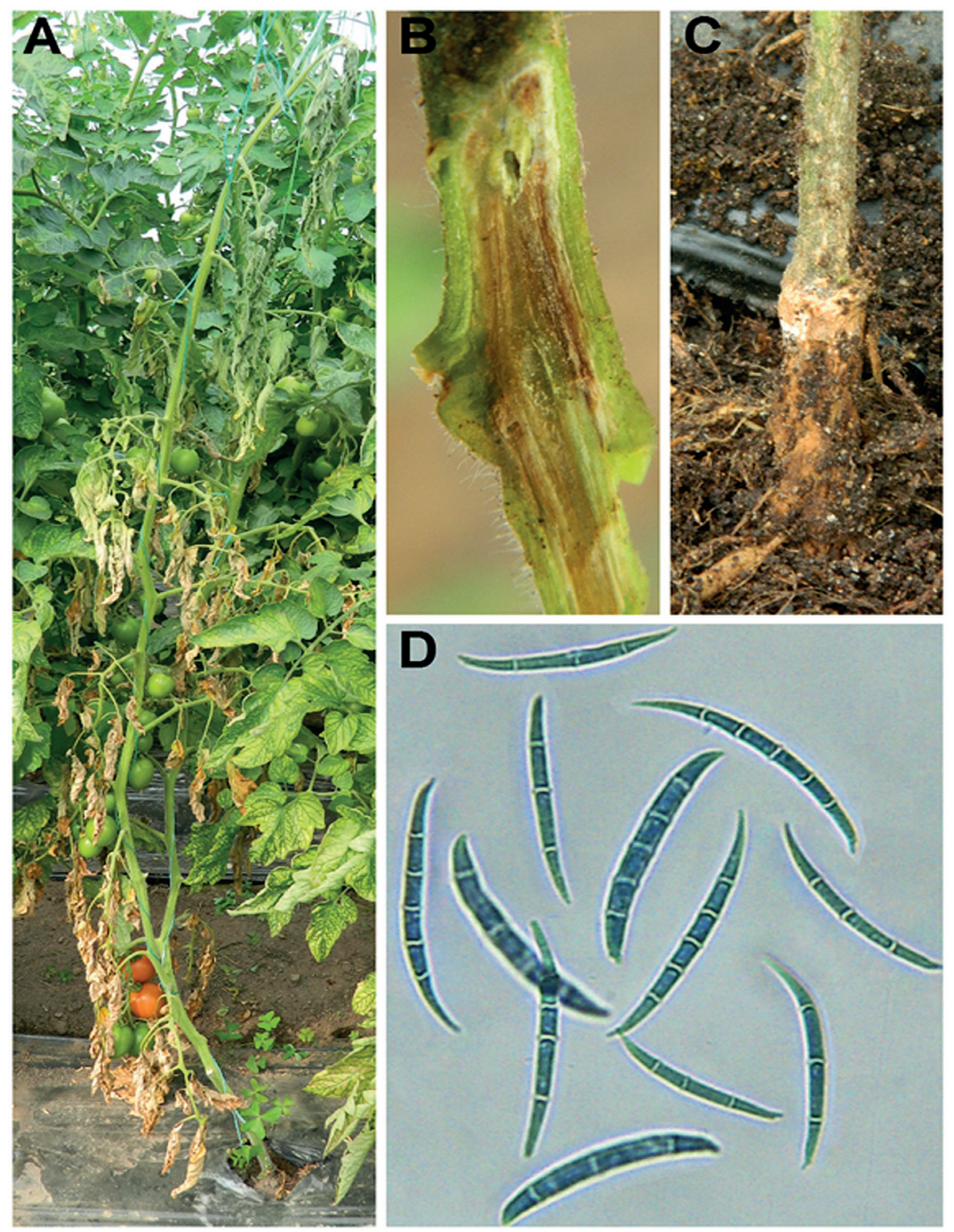

Figura 1. A. Síntoma del marchitamiento vascular causado por Fusarium oxysporum f. sp. lycopersici en una planta de tomate; B. Síntoma en detalle de la obstrucción del xilema; C. Síntoma detallado de la enfermedad en la base de una planta de tomate; $D$. Macroconidios de F. oxysporum f. sp. lycopersici. Cortesía de Juan Manuel Pineda y Agustín Calderón.

mos. La combinación de estos procesos, llamado taponamiento de los vasos por micelio, esporas, gel, gomas y tílides y el aplastamiento de los vasos por proliferación de células adyacentes de parénquima, es la responsable de la marchitez (Yadeta \& Thomma, 2013). Luego, el hongo invade todos los tejidos vegetales, alcanza la superficie externa de la planta muerta y allí esporula. Las esporas pueden ser diseminadas a nuevas plantas por el viento, el agua y así, sucesivamente.
Fusarium, se disemina a distancias cortas, mediante el agua, equipos agrícolas y herramientas infestadas por el hongo y a distancias largas, por medio de plantas enfermas o suelo adherido a ellas. Una vez el suelo es infestado, permanece así indefinidamente (Dixon \& Tilston, 2010).

Debido a que el hongo se establece como endófito en células y tejidos vasculares, se dificulta su control (Hossain et al. 2013), lo que sugiere un manejo integrado, que permita 
reducir la población del patógeno y obtener los rendimientos necesarios para tener un cultivo rentable.

Dentro de las estrategias de manejo están la aplicación de fumigantes, como $\mathrm{N}$-metil ditiocarbamato de sodio, desinfección de semilla con ácido clorhídrico al 1\%, uso de cultivares resistentes, como Torrano, Débora Max F1, Débora Plus F1, Calima y Carguero, entre otros y prácticas culturales, como embolsado conteniendo suelo esterilizado, solarización, rotación de cultivos y desinfestación de estacones.

La desinfestación del suelo con bromuro de metilo era la practica más común hasta que su uso fue proscrito, debido a que, en 1993, el Protocolo de Montreal clasificó a este producto como degradador del ozono clase I, ordenando su retiro del mercado, en el 2005 (Ministerio de Ambiente, Vivienda y Desarrollo Rural, 2006).

El uso de variedades resistentes reduce la incidencia de la enfermedad; sin embargo, la emergencia de nuevas razas del hongo y de nuevos biotipos dentro de las razas, supera su resistencia, conllevando a la búsqueda de nuevas estrategias (Horinouchi et al. 2011).

Se han identificado tres razas del hongo, conocidas como razas 1,2 y 3 o 0,1 y 2, las cuales, se han identificado en los loci: I-1, I-2 e I-3, confiriendo resistencia al patógeno, a través de genes dominantes (Scott et al. 2004; Panthee \& Chen, 2010). Según Inami et al. (2012), la raza 2 de $F$. oxysporum f. sp. lycopersici emergió de la raza 1, por la pérdida del gen avr 1 o a través de la pérdida de la función del gen, por la inserción de un trasposón, mientras que la raza 3 emergió cuando un punto de mutación ocurrió en el gen avr2. Diferentes razas del hongo portan en varias combinaciones tres genes de avirulencia, a saber: avr1, avr2 y avr3, que activan respuestas de defensa contra el hongo, al ser reconocidos por los correspondientes genes de resistencia en tomate.

La presión por prácticas sostenibles transversales a los organismos reguladores y el creciente problema de la resistencia a los plaguicidas, están obligando a hallar alternativas a los agroquímicos. El control biológico, se está utilizando en nichos específicos de la agricultura y puede ser efectivo, si se tiene una comprensión clara del agroecosistema y sus limitaciones; un ejemplo son las bacterias promotoras de crecimiento vegetal que tienen gran potencial, porque además de promover el crecimiento de las plantas, reducen la severidad de enfermedades e inducen mecanismos de resistencia (Antoun, 2013).

La Fitoepidemiología, se ha convertido en una estrategia para el manejo sostenible de los agroecosistemas. La sos- tenibilidad no implica necesariamente bajos insumos, sino el uso óptimo de los mismos. El control de las enfermedades, por ejemplo, depende en gran parte de las prácticas agrícolas, que reduzcan la incidencia o severidad de una enfermedad (Cook \& Yarham, 2006). El manejo integrado de la marchitez vascular del tomate involucra la aplicación de principios epidemiológicos y puede ser visto como la aplicación práctica de los conceptos expresados por Van der Plank (1963): $X=X_{0} \mathrm{e}^{\mathrm{rt}}$, en donde $\mathrm{X}=$ enfermedad; $\mathrm{X}_{0}=$ inóculo inicial; $\mathrm{e}=$ constante universal $(2,718281) ; \mathrm{r}=$ tasa de desarrollo de la enfermedad; $y \mathrm{t}=$ tiempo.

Esta revisión, se realizó con el objetivo de reunir la información más relevante en el ámbito nacional e internacional en relación con el manejo integrado de $F$. oxysporum $\mathrm{f}$. sp. lycopersici, indicando los factores epidemiológicos, que pueden afectar a cada una de las prácticas de manejo, según el modelo logístico de Van der Plank. Asimismo, enfatizar en la integración de las prácticas de manejo indicadas, que conlleven a reducir aplicaciones indiscriminadas de productos de síntesis química.

\section{MATERIALES Y MÉTODOS}

Se consultaron las bases de datos Scopus, Science Direct, Springerlink, Annual Reviews, Scielo y Google Académico, FAOSTAT, al igual que artículos científicos y libros. Además, se revisaron obras clásicas de las décadas del 60 y 70, como: "Plant diseases: epidemics and control", de Van der Plank (1963) y "The Genus Fusarium", de Booth (1971), hasta la información más reciente sobre el tema, como: "Integrated management strategies for tomato Fusarium wilt", de Ajilogba \& Babalola (2013); "Management of tomato diseases caused by Fusarium oxysporum", de Mc Govern (2015); "Management strategies of Fusarium wilt disease of tomato incited by Fusarium oxysporum f. sp. lycopersici (Sacc.)", de Bawa (2016) y Fusarium oxysporum and the Fusarium wilt syndrome (Gordon, 2017). Las palabras clave utilizadas fueron: Fusarium oxysporum f. sp. lycopersici, marchitez vascular, manejo integrado de Fusarium, tomate. Se incluyeron artículos de revisión, notas técnicas, estudios experimentales, publicados en idioma inglés y español.

\section{RESULTADOS Y DISCUSIÓN}

Diseminación y sobrevivencia del hongo: F. oxysporum f. sp. lycopersici se puede diseminar a través del agua (Xu et al. 2006), por esta razón, el riego por gravedad aumenta la incidencia de la marchitez vascular y la tasa de desarrollo de la enfermedad (r) (Castaño-Zapata, 2002). Asimismo, puede hacerlo a través de semilla (Mc Govern, 2015), aumentando el inóculo inicial (Xo) (Castaño-Zapata, 2002). 
El hongo también puede sobrevivir y diseminarse por medio de herramientas, maquinaria y la infraestructura usada para el cultivo, invernaderos y postes de tutorado, que sirven como reservorio de inóculo inicial (Weststeijn, 1973; Mc Govern, 2015; Neshev, 2008).

Insectos, como la mosca de las riberas [Scatella stagnalis (Diptera, Ephydridae)] pueden diseminar a F. oxysporum f. sp. lycopersici y f. sp. radicis-lycopersici (Corbaz \& Fisher, 1994; Van Eppenhuijsen et al. 2001). Se ha demostrado que Meloidogyne incognita puede causar pérdida de resistencia en tomate a la raza $1 \mathrm{de} F$. oxysporum f. sp. lycopersici (Mc Govern, 2015). Si Meloidogyne incognita ataca simultáneamente o antes del ataque de Fusarium, no afecta la resistencia a las razas 1 o 2 (Jones et al. 1976). Abawi \& Barker (1984), indican que la resistencia a la raza 1 no es afectada por este nematodo, pero sí hay un daño aditivo de los dos patógenos en variedades susceptibles de tomate, como como Bonny best.

Manejo integrado de la marchitez vascular: Los principales componentes del MIP son: prevención, monitoreo, diagnóstico correcto de la enfermedad, desarrollo de umbrales de daño económico y selección óptima de las estrategias de manejo. La prevención y el manejo comprenden una serie de prácticas, que serán explicadas a continuación.

Culturales: Incluyen prácticas que ayudan a reducir la incidencia y la severidad de la enfermedad. Son principalmente preventivas y un buen conocimiento de la naturaleza, comportamiento y condiciones ambientales de crecimiento del hongo es esencial. Dentro de este control está el uso de prácticas que conduzcan a un ambiente desfavorable para Fusarium (Ajilogba \& Babalola, 2013); por ejemplo, subir el $\mathrm{pH}$ entre 6,5 y 7,5 y emplear una fuente de nitrógeno en forma de nitrato en lugar de amoniacal (Jones et al. 1982) y la siembra de plántulas en eras elevadas, mejoran el drenaje, afectando adversamente la tasa de desarrollo de la enfermedad (r) (Castaño-Zapata, 2002). El arado profundo mejora las características físicas del suelo, modifica la porosidad del suelo, aumenta la retención de agua, la oxigenación y temperatura, acelera la descomposición de residuos vegetales al reducir su tamaño inactivando parte del inóculo presente en ellos y, en consecuencia, disminuyendo la incidencia de la enfermedad (Neshev, 2008). La población del hongo también puede ser reducida mediante cultivos de cobertura, usados como fuente de carbono (Butler et al. 2012b).

La rotación de cultivos es recomendable y se debe hacer por un periodo de 5 a 7 años (Jones et al. 1982). Esta práctica reduce el inóculo inicial $\left(\mathrm{X}_{0}\right) \mathrm{y}$, por consiguiente, la incidencia de la enfermedad (Castaño-Zapata, 2002). Es de resaltar que se debe escoger con cuidado el cultivo con el cual se va a rotar, evitando especies de la familia Solanaceae. El descan- so del terreno tiene un efecto semejante al de la rotación, reduciendo el inóculo inicial. Esta práctica es más efectiva cuando la temperatura del suelo es alta, por lo que se recomienda realizarla en épocas de verano. El suelo se debe mantener libre de plantas y ararse frecuentemente en seco, para ayudar a destruir la población del hongo (Ajilogba \& Babalola, 2013).

Se debe mantener la sanidad del cultivo, retirando plantas enfermas, residuos de podas, frutos enfermos y residuos de cosecha, junto con arvenses y sus residuos, especialmente solanáceas. Su destrucción es crucial, debido a que reducen la fuente de inóculo inicial. Las herramientas se deben limpiar y desinfestar. El empleo de ropa y de botas desinfestadas durante las labores de campo ayudan a prevenir el transporte de suelo infestado con el hongo, de un sitio a otro (Neshev, 2008).

Heridas hechas a las plantas durante el tutorado, raleo, poda y plateo, aumentan la susceptibilidad a la enfermedad, mediante el incremento de la tasa de desarrollo (Ajilogba \& Babalola, 2013).

Desarrollo de variedades resistentes: La resistencia genética es el método más eficiente, económico y ecológico de manejo de la enfermedad, pero las interacciones planta-patógeno están lejos de ser simples. La analogía de una carrera armamentista se ha usado para explicar la coevolución de las plantas y sus patógenos; las plantas desarrollan mecanismos, los patógenos desarrollan estrategias para vencer la resistencia de las plantas, las plantas sucesivamente desarrollan nuevas tácticas de defensa, las cuales, seleccionan para más cambios en el patógeno.

Hay dos tipos de resistencia: la poligénica, horizontal o de genes menores, que no reconoce razas específicas del patógeno y la vertical, monogénica o de genes mayores, que reconoce razas específicas e imparte un alto nivel de resistencia. La resistencia poligénica, generalmente, se basa en múltiples genes, que actúan para crear barreras físicas o químicas que impiden la invasión del patógeno. Se ha identificado este tipo de resistencia al hongo en variedades de tomate, como Homestead y Marglobe, pero no han sido de interés para el desarrollo de variedades comerciales, con este tipo de resistencia (Gao et al. 1995). La resistencia poligénica es más estable y afecta la tasa de desarrollo de la enfermedad (r), mientras que la resistencia vertical, afecta el inóculo inicial (Xo) (Castaño-Zapata, 2002).

Las variedades de tomate resistentes a las razas 1 y 3 de $F$. oxysporum f. sp. lycopersici han sido mejoradas, mediante la introgresión de genes de sus parientes silvestres, como Solanum pennellii y $S$. pimpinellifollium. Muchas variedades de tomate mejoradas convencionalmente tienen resistencia 
a las razas 1 o 2; varias también poseen una combinación de resistencia a las razas 1 y 2; pocas poseen resistencia a todas las razas del hongo, reportadas hasta ahora (Mc Govern, 2015).

Modificación genética: Se ha avanzado en la modificación genética del tomate contra el hongo, usando genes derivados de otras especies; por ejemplo, se han utilizado genes de Medicago sativa y Wasabia japonica mostrando mayor resistencia a la enfermedad, que las plantas no transformadas (Abdallah et al. 2010). Líneas transgénicas, a partir de quitinasa de trigo, expresan alto nivel resistencia al hongo, siendo el rasgo heredable para la primera y la segunda generación (Girhepuje \& Shinde, 2011). También, se ha demostrado que la transformación del tomate con quitinasa derivada de arroz reduce significativamente la incidencia de la marchitez vascular (Abdallah et al. 2010).

Resistencia inducida: La resistencia sistémica adquirida RSA- y resistencia sistémica inducida -RSI- han sido bien estudiadas. La RSA es inducida por la exposición de una planta a elicitores abióticos (químicos) o bióticos (microorganismos patogénicos o no patogénicos), es dependiente de la producción de ácido salićlico y está asociada con la acumulación de proteínas relacionadas con la patogénesis, PR; por otra parte, la RSI es activada por la exposición de las raíces a aislamientos específicos de BPCV, en particular Bacillus spp. y Pseudomonas spp. (Vallad \& Goodman, 2004).

Injertación: Es el uso de injertos tienen como principal ventaja que no contaminan al ambiente, además, incrementan la tolerancia a temperaturas altas y bajas, a la salinidad del suelo o agua y a sequía; asimismo, aumenta el vigor de las plantas y el rendimiento, como resultado de mayor absorción de nutrientes y contenido mineral en la parte aérea. Esta tecnología ha sido exitosa en tomate, pimiento, sandía, melón y berenjena (INTAGRI, 2017). El empleo de tomate injertado se ha acelerado, por la prohibición del bromuro de metilo, pero la adopción de esta práctica ha sido lenta, debido a la percepción de su alto costo (Mc Govern, 2015).

Control químico: Existen diferentes tipos de químicos que ayudan a prevenir o a manejar a la enfermedad. A continuación, se describen los diferentes químicos usados:

Desinfestantes: Debido a la gran capacidad de supervivencia del hongo en diferentes sustratos, incluyendo semillas de tomate, agua de riego, recipientes, estacas y estructuras de soporte, la eliminación de los propágulos de Fusarium, a través de la desinfestación, es esencial para su manejo (Agrios, 2005). Se usan compuestos oxidantes fuertes, como el NaO$\mathrm{Cl}, \mathrm{H}_{2} \mathrm{O}_{2}$ y $\mathrm{O}_{3}$, que inactivan el patógeno, por medio de la ruptura de los ácidos nucleicos. Otros desinfectantes, como los alcoholes y las sales cuaternarias de amonio, causan de- naturación de proteínas y ruptura de las membranas celulares (Mc Govern, 2015). El formaldehído mata los macroconidios del hongo depositados en las estructuras y paredes de los invernaderos (Weststeijn, 1973).

Fungicidas: Aunque hay fungicidas efectivos contra el hongo, se usan con menos frecuencia que los fumigantes y otras estrategias de manejo, excepto en sistemas bajo invernadero. Se ha demostrado que bromuconazole y procloraz, cuando se aplican al suelo, son más efectivos en reducir la incidencia de la marchitez vascular, que azoxystrobin, benomil, carbendazim y fluidioxonil (Amini \& Sidovich, 2010), afectando la tasa de desarrollo (r) de la enfermedad (Castaño-Zapata, 2002). El uso indiscriminado de fungicidas puede causar el desarrollo de nuevas cepas patogénicas resistentes, la reducción de especies benéficas, problemas de salud en los operarios, acumulación de residuos en el suelo, fuentes de agua y alimentos (Castro \& Ramos, 2004). Actualmente, se emplean productos de poca toxicidad, pero su uso inadecuado puede causar daños severos al ambiente y a los seres vivos; además, se requiere tomar precauciones, para evitar toxicidad en las plantas (INTAGRI, 2017).

Fumigantes: Después de la resistencia genética, la fumigación en presiembra ha sido la estrategia de manejo más común para controlar la marchitez vascular, afectando el inóculo inicial $\left(\mathrm{X}_{0}\right)$ (Castaño-Zapata, 2002). El bromuro de metilo en combinación con tricloronitrometano ha sido el tratamiento estándar para controlar esta y otras enfermedades causadas por patógenos, cuyo hábitat es el suelo; sin embargo, como se mencionó previamente, el bromuro de metilo ha sido eliminado en conformidad con el Protocolo de Montreal (Watson et al. 1992). Otras sustancias, como dicloropropeneb, $\beta$ cloropicrin, cloropicrin, metil isotiocianato, entre otras, también reducen la incidencia de la enfermedad (Mc Govern, 2015).

Desinfestación anaeróbica del suelo: La aplicación de materia orgánica seguida por irrigación y cobertura del suelo con polietileno crean condiciones de anaerobiosis (Mc Govern, 2015). La reducción de la población de patógenos habitantes del suelo, como Fusarium, se atribuye a condiciones anaeróbicas, temperaturas altas, generación de ácidos orgánicos, acumulación de compuestos volátiles y liberación de iones metálicos (Mc Govern, 2015).

Nutrición vegetal y química del suelo: La nutrición de las plantas puede influenciar su susceptibilidad a enfermedades (Datnoff et al. 2007), afectando la tasa de desarrollo (r) (Castaño-Zapata, 2002). El hidróxido de calcio reduce la incidencia y la tasa de desarrollo de la marchitez vascular y se atribuye su efecto al aumento del $\mathrm{pH}$ del suelo $(7,5-8,0)$ y no a la acumulación de calcio. El incremento del $\mathrm{pH}$ reduce la disponibilidad de micronutrientes, como el hierro, mangane- 
so y zinc, esenciales para el hongo. Se sugiere que Fusarium es más sensible a baja disponibilidad de nutrientes que las plantas de tomate, y que esta es la base de su manejo, a través de nutrición. La ceniza, rica en dióxido de silicio y de calcio, también disminuye la población del hongo.

Control biológico: Últimamente, ha crecido el interés sobre el manejo del hongo a través del control biológico (Mc Spadden et al. 2002). Los biocontroladores incluyen bacterias, como Pseudomonas fluorescens y Burkholderia cepacia y hongos, como Trichoderma spp., Gliocladium virens y aislamientos no patogénicos de Fusarium spp. Hay formulaciones comerciales a base de Gliocladium virens (SoilGard $($ ) y Trichoderma harzianum (RootShield $($ ), altamente efectivas para controlar al hongo (Larkin \& Fravel, 1998). Las interacciones que ocurren entre los biocontroladores y patógenos son complejas, como son la interacción de la microbiota del suelo, en general, y puede involucrar antibiosis, competencia por nutrientes, en especial, hierro, a través de la producción de sideróforos y sitios de colonización, inducción de resistencia, hiperparasitismo y predación (Pal \& Mc Spadden, 2006).

El manejo de la marchitez vascular abarca un amplio rango de microorganismos solos o en combinación, en donde se incluyen diferentes aislamientos de la misma especie bacteriana, diferentes géneros de bacterias y de hongos y mezclas de éstos. Varios biocontroladores reducen la producción de esporas, la germinación y la supervivencia de $F$. oxysporum f. sp. Lycopersici, por antibiosis, competencia y resistencia inducida. En general, los biocontroladores disminuyen significativamente la marchitez vascular y, en algunos casos, pueden ser superiores al efecto de los fungicidas. En la mayoría de los casos, las combinaciones de biocontroladores son más efectivas que sus componentes individuales, lo que sugiere que el incremento en la diversidad microbiana es benéfico en el manejo de la enfermedad (Mc Govern, 2015).

Control físico: Las técnicas que usan calor para inactivar o debilitar a los patógenos incluyen vapor, solarización y compostaje (Mc Govern, 2015), afectando el inóculo inicial ( $\left.\mathbf{X}_{\circ}\right)$ (Castaño-Zapata, 2002). Las últimas dos técnicas también afectan a los patógenos, mediante el aumento de microorganismos antagonistas o benéficos e incremento de nutrientes, disponibles para las plantas (Mc Govern, 2015).

El vapor fue el desinfestante de suelo más común en cultivos de hortalizas y ornamentales de alto valor que crecían bajo invernadero; sin embargo, el alto costo de los combustibles condujo a los agricultores a utilizar otros métodos, como los fumigantes de menor costo. El vapor aireado es preferible, debido a que reduce las densidades del patógeno a temperaturas más bajas $\left(60\right.$ a $\left.70^{\circ} \mathrm{C} / 30 \mathrm{~min}\right)$, que las del vapor no aireado (cerca de $100^{\circ} \mathrm{C}$ ) y evita la eliminación total de microorganismos benéficos (Mc Govern, 2015). Según Weststeijn (1973), el empleo de vapor a $80^{\circ} \mathrm{C}$ durante $12 \mathrm{~h}$ bajo cubiertas de lona en invernadero es tan efectivo como cloropicrina y bromuro de metilo en la reducción de la marchitez vascular.

La solarización puede reducir la densidad del patógeno, mediante la inactivación térmica, el incremento de microorganismos antagónicos termofilicos y termotolerantes, acumulación de compuestos volátiles y cambios en la composición de gases del suelo (Mc Govern, 2015). Una de las limitaciones de la solarización es que sus mayores efectos no superan los 30 cm de profundidad del suelo (Mc Govern \& Mc Sorley, 2012). La solarización del suelo durante 8 semanas puede reducir la población del hongo, entre 91 y $98 \%$, siendo tan efectiva como el bromuro de metilo, aumentando significativamente el rendimiento del cultivo del tomate (Ioannou, 2000).

El compostaje involucra la degradación microbiana controlada de materia orgánica y es más común de forma aeróbica. Además de calor, el compostaje aeróbico produce amonio, dióxido de carbono y agua, mientras la descomposición anaeróbica produce $\mathrm{CH}_{4}, \mathrm{CO}_{2}$ y muchos compuestos orgánicos intermedios (Bernal et al. 2009). A menos que sea controlado por liberación deliberada de calor, a través de ventilación, la temperatura del compostaje suele ser a $80^{\circ} \mathrm{C}$; sin embargo, la actividad microbiana para la descomposición del sustrato requiere que la temperatura del compostaje sea mantenida por debajo de $60^{\circ} \mathrm{C}$ (Mc Govern, 2015).

Tratamiento del agua: Las técnicas físicas para desinfestación del agua de riego recirculada y soluciones nutritivas, incluyen filtración, calor y radiación UV; por ejemplo, la filtración del agua para la remoción del patógeno o inóculo inicial puede involucrar baja percolación, a través de materiales, como arena fina, lana de roca, entre otros o movimiento más rápido, por medio de membranas (ultrafiltración). También la filtración lenta tiene un componente biológico y un biofilm microbial, que se forma sobre la superficie del material de filtración, siendo incompatible con la desinfestación química. Si el agua contiene grandes cantidades de arcilla y de sólidos solubles, ambos sistemas requieren un paso de prefiltración.

La mayoría de patógenos de plantas, incluyendo a $F$. oxysporum, pueden morir a $54^{\circ} \mathrm{C}$ por 15 seg (Runia \& Amsing, 2001); por consiguiente, la pasteurización se convierte en una opción para matar los conidios del hongo, requiriendo que el agua o solución nutritiva sea calentada a $95^{\circ} \mathrm{C}$ por 30 min (Newman, 2004).

\section{Combinación de prácticas:}

Biológicas y culturales: Bacillus subtilis CRB20 en combinación con quitina promueve el crecimiento de las plantas y 
la supresión de F. oxysporum f. sp. lycopersici (Hariprasad et al. 2011). Asimismo, la combinación de la desinfestación anaeróbica del suelo con solarización reduce, en mayor grado, la sobrevivencia del hongo, si se compara con el bromuro de metilo (Butler et al. 2012a). Pseudomonas spp., Trichoderma harzianum y Glomus intraradices tienen la capacidad de reducir hasta en un $67 \%$ la incidencia de la marchitez vascular, reducción que se puede incrementar hasta un74\%, cuando se añade una enmienda orgánica (Srivastava et al. 2010).

La combinación de micorizas arbusculares y cultivos intercalados, como el puerro [Allium ampeloprasum var. porrum (L.) J.Gay], también disminuye la incidencia de la marchitez vascular del tomate (Hage-Ahmed et al. 2013).

El sustrato obtenido del compostaje de orujo de uva (Vitis vinífera L.) puede reducir el área bajo la curva del desarrollo de la marchitez, en un $92 \%$. Este sustrato tiene la característica de subir el $\mathrm{pH}$ hasta 7,9, acompañado de una alta actividad de $\beta$-glucosidasa $\left(110,04 \mu \mathrm{g}\right.$ de $\mathrm{p}$-nitrofenol $\left./ \mathrm{cm}^{3}\right)$, condiciones adversas para F. oxysporum f. sp. lycopersici (Borrero et al. 2004).

Biológicas y químicas: La aplicación de bacterias promotoras de crecimiento vegetal, como Pseudomonas fluorescens en combinación con una dosis reducida de benomil es más efectiva en la reducción del área bajo la curva de la marchitez vascular del tomate (Someya et al. 2006). Igual resultado se ha obtenido con Azotobacter sp., Bacillus cereus y B. megaterium, en combinación con ácido húmico (Abdel-Monaim et al. 2012).
Culturales y físicas: El uso de vapor caliente para desinfestar el suelo combinado con la aplicación de formaldehído en las estructuras de los invernaderos puede inhibir por completo la germinación de los macroconidios del hongo. Otras prácticas, como la desinfestación de tuberías surtidoras de riego, la eliminación de plantas enfermas, desinfestación de sitios y equipos de trabajo infestados con el patógeno y el uso de material vegetal sano cuando se establece un cultivo de tomate, son muy eficientes para el manejo de la enfermedad (Weststeijn, 1973).

El uso de enmiendas orgánicas reduce la incidencia de la marchitez vascular y la siembra de tomate con franjas de pasto bahía (Paspalum notatum Fluguee), es una práctica favorable para suprimir al hongo. Asimismo, la rotación de tomate con cultivos de $P$. notatum durante 4 años puede disminuir hasta un $6 \%$ la incidencia de la enfermedad, a través de la destrucción de las estructuras reproductivas del hongo (Chellemi et al. 2012).

Efecto epidemiológico de las prácticas de manejo: El control de enfermedades involucra la aplicación práctica de medidas expresadas, mediante el modelo logístico de Van der Plank (1963): $\mathrm{X}=\mathrm{X}_{0} \mathrm{e}^{\mathrm{rt}}$. El manejo de la enfermedad consiste en retardar el desarrollo de la epidemia, a través de la interacción de varias prácticas. Este retardo depende de la reducción de inóculo inicial $\left(\mathbf{X}_{0}\right)$, de la tasa de desarrollo de la enfermedad (r) y tiempo (t) (Castaño-Zapata, 2002; Cook \& Yarham, 2006), como se ha visto en esta revisión y se resume en la tabla 1.

Tabla 1. Prácticas de manejo de la marchitez vascular del tomate y su efecto sobre cada uno de los parámetros del modelo logístico de Van der Plank.

\begin{tabular}{|c|c|}
\hline ESTRATEGIAS & FACTOR EPIDEMIOLÓGICO \\
\hline \multicolumn{2}{|c|}{ PRÁCTICAS CULTURALES } \\
\hline Rotación de cultivos & $\mathrm{X}_{0}$ \\
\hline Sanidad & $\mathrm{X}_{0}$ \\
\hline Fertilización & $\mathrm{r}$ \\
\hline Encalado & $\mathrm{r}$ \\
\hline Solarización & $\mathrm{X}_{0}$ \\
\hline Barbecho & $\mathrm{X}_{0}$ \\
\hline Coberturas & $\mathrm{r}$ \\
\hline Riego & $\mathrm{r}$ \\
\hline Manejo de arvenses & $\mathrm{X}_{0}$ \\
\hline Siembra en camas elevadas & $\mathrm{r}$ \\
\hline Época de siembra y cosecha & $\mathrm{t}$ \\
\hline Cultivos intercalados & $\mathrm{X}_{0}$ \\
\hline
\end{tabular}


Continuación Tabla 1.

\begin{tabular}{|l|c|}
\hline \multicolumn{2}{|c|}{ CONTROL GENÉTICO } \\
\hline Resistencia monogénica & $\mathrm{X}_{0}$ \\
\hline Resistencia poligénica & $\mathrm{r}$ \\
\hline Modificación genética & $\mathrm{X}_{0}$ \\
\hline Resistencia sistémica & $\mathrm{r}$ \\
\hline Injertación & $\mathrm{X}_{0}$ \\
\hline \multicolumn{2}{|c|}{ CONTROL BIOLÓGICO } \\
\hline Hongos & $\mathrm{X}_{0}$ \\
\hline Bacterias & $\mathrm{X}_{0}$ \\
\hline $\begin{array}{l}\text { Bacterias promotoras de crecimien- } \\
\text { to vegetal }\end{array}$ & $\mathrm{r}$ \\
\hline \multicolumn{2}{|c|}{ CONTROL QUÍMICO } \\
\hline Desinfestantes & $\mathrm{X}_{0}$ \\
\hline Fungicidas & $\mathrm{r}$ \\
\hline Fumigantes & $\mathrm{X}_{0}$ \\
\hline
\end{tabular}

\section{CONCLUSIONES Y RECOMENDACIONES}

Aunque el control más efectivo de la enfermedad ha sido mediante la aplicación de productos de síntesis química, causando problemas de resistencia en el hongo, intoxicación de los operarios y contaminación del medio ambiente, se puede evitar, integrándolos con otras prácticas. El manejo efectivo de la marchitez vascular solo se puede lograr, a través del empleo de diferentes prácticas que incidan sobre los factores que determinan el desarrollo de una epidemia y enfocadas dentro del modelo logístico de Van der Plank. Se debe priorizar la investigación en el desarrollo de materiales resistentes, como estrategia más efectiva y económica dentro del manejo integrado de la marchitez vascular.

Conflicto de intereses: El manuscrito fue preparado y revisado con la participación de todos los autores, quienes declaramos que no existe conflicto de intereses que ponga en riesgo la validez de los resultados presentados.

\section{BIBLIOGRAFÍA}

1. ABAWI, G.S.; BARKER, K.R. 1984. Effects of cultivar, soil temperature, and levels of Meloidogyne incognita on root necrosis and Fusarium wilt of tomatoes. Phytopathology. 7:433-438.

2. ABDALLAH, N.A.; SHAH, D.; ABBAS, D.; MADKOUR, M. 2010. Stable integration and expression of a plant defensin in tomato confers resistance to Fusarium wilt. GM Crops. 1(5):344-350.
3. ABDEL-MONAIM, M.F.; ABDEL-GAID, M.A.; EL-MORSY, M.; EL-MORSY, A. 2012. Efficacy of rhizobacteria and humic acid for controlling Fusarium wilt disease and improvement of plant growth, quantitative and qualitative parameters in tomato. Sci J. Plant Pathol. 1:39-48.

4. AGRIOS, G.N. 2005. Plant pathology. Fifth Ed. Academic Press, Burlington. 635p.

5. AJILOGBA, C.F.; BABALOLA, O.O. 2013. Integrated management strategies for tomato Fusarium wilt. Biocontrol Science. 18(3):117-127.

6. AMINI, J.; SIDOVICH, D.F. 2010. The effects of fungicides on Fusarium oxysporum f. sp. lycopersici associated with Fusarium wilt of tomato. J. Plant Prot. Res. 50:172-178.

7. ANTOUN, H. 2013. Plant-growth-promoting rhizobacteria. In: Maloy, S.; Hughes K. (eds.). Brenner's Encyclopedia of Genetics (Second Edition). Academic Press, San Diego. p.353-355.

8. BAWA, I. 2016. Management strategies of Fusarium wilt disease of tomato incited by Fusarium oxysporum f. sp. lycopersici (Sacc.): A review. Int. J. of Advanced Academic Research. 2(5):32-42.

9. BERNAL, M.P.; ALBURQUERQUE, J.A.; MORAL, R. 2009. Composting of animal manures and chemical criteria for compost maturity assessment. A review. Bioresource Technology. 100(22):5444-5453. 
10. BOOTH, C. 1971. The genus Fusarium. Published by Commonwealth Agricultural Bureaux, for the Commonwealth Mycological Institute. Surrey, Inglaterra. 237p.

11. BORRERO, C.; TRILLAS, M.I.; ORDOVÁS, J.; TELLO, J.C.; AVILÉS, M. 2004. Predictive factors for the suppression of Fusarium wilt of tomato in plant growth media. Phytopathology. 94(10):1094-1101.

12. BUTLER, D.M.; KOKALS-BURELLE, N.; MURAMOTO, J.; SHENNAN, C.; MC COLLUM, T.G.; ROSSKOPF, E.N. 2012a. Impact of anaerobic soil disinfestation combined with soil solarization on plant-parasitic nematodes and introduced inoculum of soilborne plant pathogens in raised-bed vegetable production. Crop Protection. 39:33-40.

13. BUTLER, D.M.; ROSSKOPF, E.N.; KOKALIS-BURELLE, N.; ALBANO, J.P.; MURAMOTO, J.; SHENNAN, C. 2012b. Exploring warm-season cover crops as carbon sources for anaerobic soil disinfestation (ASD). Plant Soil. 355:149-165.

14. CASTAÑO-ZAPATA, J. 2002. Principios básicos de fitoepidemiología. Editorial Universidad de Caldas (Colombia). 396p.

15. CASTRO, P.A.; RAMOS, J.P. 2005. Determinación de residuos de plaguicidas organofosforados en muestras de tomate de la ciudad de Bogotá (Universidad de los Andes,Bogotá, Colombia). Disponible desde Internet en: http://www.guaica.uniandes.edu.co:5050/ dspace/bitstre am/1992/661/1/mi_1106.pdf (con acceso 2410/2017).

16. CHELLEMI, D.O.; WU, T.; GRAHAM, J.H.; CHURCH, G. 2012. Biological impact of divergent land management practices on tomato crop health. Phytopathology. 102(6):597-608.

17. CORBAZ, R.; FISCHER, S. 1994. The shore fly Scatella stagnalis Fall. Diptera, Ephydridae responsible for transmitting Fusarium oxysporum f. sp. lycopersici in soilless tomato crops. Rev. Suisse Vitic. Arboric. Hortic. 26(6):383-385.

18. COOK, R.J.; YARHAM, D.J. 2006. Epidemiology in sustainable systems. In: Cooke, B.M.; Jones, D.G.; Kaye, B. (Eds.). The epidemiology of plant diseases. Dordrecht: Springer. p.309-334.
19. DATNOFF, L.E.; ELMER, W.H.; HUBER, D.M. 2007. Mineral nutrition and plant disease. APS Press, St. Paul, MN. 278p.

20. DIXON, G.R.; TILSTON, E.L. 2010. Soil-borne pathogens and their interactions with the soil environment. In: Dixon, G.R.; Tilston, E.L. (Eds) Soil microbiology and sustainable crop production. Ed. Springer. (Netherland): p.197-271.

21. ENESPA ; DWIVEDI, S.K. 2014. Effectiveness of some Antagonistic fungi and botanicals against Fusarium solani and Fusarium oxysporum f. sp. lycopersici infecting brinjal and tomato plants. Asian J. Plant Path. 8(1):18-25.

22. FAOSTAT. 2014. Statistical Databases. Food and Agriculture Organization of the United Nations. Roma. Italia. Disponible desde Internet en: http://www.fao. org/faostat/es/\#data/QC (con acceso 27/06/2017).

23. FASSIHIANI, A. 2000. Symptomless carriers of the causal agent of tomato wilt pathogen. J. Agric. Sci. Tech. 2:27-32.

24. GAO, H.; BECKMAN, C.H.; MUELLER, W.C. 1995. The nature of tolerance to Fusarium oxysporum f. sp. lycopersici in polygenically field-resistant marglobe tomato plants. Physiol. Mol. Plant Pathol. 46:401412.

25. GIRHEPUJE, P.V.; SHINDE, G.B. 2011. Transgenic tomato plants expressing a wheat endochitinase gene demonstrate enhanced resistance to Fusarium oxysporum f. sp. lycopersici. Plant Cell Tiss. Organ Cult. 105:243-251.

26. GORDON, T.R. 2017. Fusarium oxysporum and the Fusarium wilt syndrome. Ann. Rev. Phytopathology. 55:23-39.

27. HAGE-AHMED, K.; KRAMMER, J.; STEINKELLNER, S. 2013. The intercropping partner affects arbuscular mycorrhizal fungi and Fusarium oxysporum f. sp. lycopersici interactions in tomato. Mycorrhiza. 23(7):543-550.

28. HARIPRASAD, P.; DIVAKARA, S.T.; NIRANJANA, S.R. 2011. Isolation and characterization of chitinolytic rhizobacteria for the management of Fusarium wilt in tomato. Crop Protection. 30(12):1606-1612.

29. HORINOUCHI, H.; WATANABE, H.; TAGUCHI, Y.; MUSLIM, A.; HYAKUMACHI, M. 2011. Biological control 
of Fusarium wilt of tomato with Fusarium equiseti GF191 in both rock wool and soil systems. Biocontrol. 56(6):915-923.

30. HOSSAIN, M.M.; HOSSAIN, N.; SULTANA, F.; ISLAM, S.M.N.; ISLAM, M.S.; BHUIYAN, M.K.A. 2013. Integrated management of Fusarium wilt of chickpea (Cicer arietinum L.) caused by Fusarium oxysporum f. sp. ciceris with microbial antagonist, botanical extract and fungicide. African J. of Biotech. 12(29):4699-4706.

31 INAMI, K.; KASHIWA, T.; KAWABE, M.; ONOKUBOOKABE, A.; ISHIKAWA, N.; PÉREZ, E.R.; MADADI, K.A. 2014. The tomato wilt fungus Fusarium oxysporum f. sp. lycopersici shares common ancestors with nonpathogenic $F$. oxysporum isolated from wild tomatoes in the Peruvian Andes. Microbes and Environments. 29(2):200-210.

32. INAMI, K.; YOSHIOKA-AKIYAMA, C.; MORITA, Y.; YAMASAKI, M.; TERAOKA, T. 2012. A Genetic mechanism for emergence of races in Fusarium oxysporum $\mathrm{f}$. sp. lycopersici: inactivation of avirulence gene AVR1 by transposon insertion. PLoS One 7 (8):e44101.

33. IOANNOU, N. 2000. Soil solarization as a substitute for methyl bromide fumigation in greenhouse tomato production in Cyprus. Phytoparasitica. 28(3):248256.

34. INTAGRI S.C. 2017. Ventajas del injerto en tomate y su proceso paso a paso Disponible desde Internet en https:/www.intagri.com/articulos/hortalizas/ injerto-en-tomate-y-procesos-paso-a-paso?utm source $=$ Sendgrid\&utm_medium $=$ Art-ventajas $\% 20$ del\%20injerto\# (con acceso el 12/10/2017).

35. JONES, J.P.; JONES, J.B.; MILLER, J.W. 1982. Fusarium wilt of tomato. Plant Pathology. Circular No237. Fla. Dep. Agric. \& Consumer Serv. Division of Plant Industry. June 1982. 2p.

36. JONES, J.P. 1991. Fusarium wilt. p. 15-16. In: Compendium of Tomato Diseases. J. B. Jones et al. (Eds.). APS PRESS. The American Phytopathological Society. 73p.

37. JONES, J.P.; OVERMAN, A.J.; CRILL, P. 1976. Failure of root-knot nematode to affect Fusarium wilt resistance in tomato. Phytopathology. 66:1339-1346.

38. KANT, P.; REINPRECHT, Y.; MARTIN, C.J.; ISLAM, R.; PAULS, K.P. 2011. Integration of biotechnologies: di- sease resistance pathology Fusarium. In: Moo-Young M. (ed.). Comprehensive Biotechnology, second edition, Elsevier, Amsterdam. p.729-743.

39. KATAN, T.; SHLEVIN, E.; KATAN, J. 1997. Sporulation of Fusarium oxysporum f. sp. lycopersici on stem surfaces of tomato plants and aerial dissemination of inoculum. Phytopathology. 87:712-719.

40. LARKIN, R.P.; FRAVEL, D.R. 1998. Efficacy of various fungal and bacterial biocontrol organisms for control of Fusarium wilt of tomato. Plant Disease 82(9):10221028.

41. MC GOVERN, R.J.; MC SORLEY, R. 2012. Management of bacterial and fungal plant pathogens by soil solarization. In: Gamliel, A., Katan, J. (Eds.), Soil Solarization: Theory and Practice. APS Press, Minneapolis, MN. p.53-62.

42. MC GOVERN, R.J. 2015. Management of tomato diseases caused by Fusarium oxysporum. Crop Protection. 73:78-92.

43. MC GOVERN, R.J.; DATNOFF, L.E., 1992. Fusarium crown and root rot of tomato:reevaluation of management strategies. In: Vavrina, C.S. (Ed.), Fla. Tom. Instit. Proc., Vegetable Crops Special Series, SS HOS 1 University of Florida-IFAS, p.75-82.

44. MC SPADDEN GARDENER, B.B.; FRAVEL, D.R. 2002. Biological control of plant pathogens: Research, commercialization, and application in the USA. Online Plant Health Prog. Disponible desde Internet en http://www.plantmanagementnetwork.org/pub/php/ review/biocontrol/ (con acceso el 27/12/2016).

45. MINISTERIO DE AMBIENTE, VIVIENDA Y DESARROLLO RURAL. 2006. Resolución 1047, por la cual se establecen medidas ambientales para la aplicación de la sustancia bromuro de metilo en tratamientos cuarentenarios en Colombia. En: Biblioteca virtual de Medio Ambiente. Disponible desde Internet en: http://www.minambiente.gov.co/images/Asuntosambientalesy SectorialyUrbana/pdf/Legislacion/ res_1047_120606.pdf (con acceso el 20/06/ 2017).

46. NEWMAN, S.E. 2004 Disinfecting irrigation water for disease management. In: $20^{\text {th }}$ Annual Conference on Pest Management on Ornamentals. p.20-22.

47. NESHEV, G. 2008. Major soil-borne phytopathogens on tomato and cucumber in Bulgaria, and methods for their management. In: Labrada, R. (ed). Alternatives 
to replace methyl bromide for soil-borne pest control in east and central Europe. FAO. p.1-22.

48. PAL, K.K.; MC. SPADDEN GARDENER, B. 2006. Biological control of plant pathogens. Plant Health Instr. 2:1117-1142.

49. PANTHEE, D.R.; CHEN, F. 2010. Genomics of fungal disease resistance in tomato. Curr. Genomics. 11:3039.

50. RAMYABHARATHI, S.A.; MEENA, B.; RAGUCHANDER, T. 2012 Induction of chitinase and $\beta$-1, 3-glucanase $\mathrm{PR}$ proteins in tomato through $\mathrm{h}$ liquid formulated Bacillus subtilis EPCO 16 against Fusarium wilt. Journal of Today's Biological Sciences: Research \& Review. India. 1(1):50-60.

51. RUNIA, W.T.; AMSING, J.J. 2001. Lethal temperatures of soilborne pathogens in recirculation water from closed cultivation systems. Acta Hortic. 554:333-339.

52. SCOTT, J.W.; AGRAMA, H.A.; JONES, J.P. 2004. RFLPbased analysis of recombination among resistance genes to Fusarium wilt races 1, 2, and 3 in tomato. J. Am. Soc. Hortic. Sci. 129 (3):394-400.

53. SMITH, S. 2007. An overview of ecological and habitat aspects in the genus Fusarium with special emphasis on the soil-borne pathogenic forms. Plant Pathol. Bull. 16:97-120.

54. SOMEYA, N.; TSUCHIYA, K.; YOSHIDA, T.; NOGUCHI, M.T.; SAWADA, H. 2006. Combined use of the biocontrol bacterium Pseudomonas fluorescens strain Irb3w1 with reduced fungicide application for the control of tomato Fusarium wilt. Biocontrol Sci. 11(2):75-80.

55. SRIVASTAVA, R.; KHALID, A.; SINGH, U.S.; SHARMA, A.K. 2010. Evaluation of arbuscular mycorrhizal fungus, fluorescent Pseudomonas and Trichoderma harzianum formulation against Fusarium oxysporum f. sp. lycopersici for the management of tomato wilt. Biological Control. 53(1):24-31.

56. VALLAD, G.E.; GOODMAN, R.M. 2004. Systemic acquired resistance and induced systemic resistance in conventional agriculture. Crop Sci. 44(6):19201934.

57. VAN DER PLANK, J.E. 1963. Plant diseases: epidemics and control. Academic Press. New York. 349p.

58. VAN EPPENHUIJSEN, C.W.; PAGE, B.B.C.; KOOLAARD, J.P. 2001. Preventative treatments for control of fungus gnats and shore flies. New Zeal. Plant Prot. 54:42-46.

59. WATSON, R.T.; ALBRITTON, D.T.; ANDERSON, S.O.; LEE-BAPTY, S. 1992. Methyl Bromide: Its atmospheric science, technology and economics. Montreal Protocol Assessment Suppl., U.N.E.P., Nairobi, Kenya. 234p.

60. WESTSTEIJN, G. 1973. Soil sterilization and glasshouse disinfection to control Fusarium oxysporum f. sp. lycopersici in tomatoes in the Netherlands. Neth. J. Plant Pathol. 79(1):36-40.

61. XU, L.; NONOMURA, T.; SUZUKI, S.; KITAGAWA, Y.; TAJIMA, H.; OKADA, K.; KUSAKARI, S; MATSUDA, Y.; TOYODA, H. 2006. Symptomatic evidence for differential root invasion by Fusarium crown and root rot pathogens between common tomato Lycopersicon esculentum and its varieties. J. Phytopathol. 154:577-586.

62. YADETA, K.A.; THOMMA, B.P. 2013. The xylem as battleground for plant hosts and vascular wilt pathogens. Frontiers in Plant Science. 4:97.

Recibido: Marzo 7 de 2017

Aceptado: Octubre 23 de 2017

Cómo citar:

Vásquez-Ramírez, L.M.; Castaño-Zapata, J. 2017. Manejo integrado de la marchitez vascular del tomate [Fusarium oxysporum f. sp. lycopersici (Sacc.) W.C. Snyder \& H.N. Hansen]: una revisión. Rev. U.D.C.A Act \& Div. Cient. 20(2): 363-374. 